\title{
High-Resolution TEM/STEM by Means of Advanced Instrumentation
}

\author{
Max. Haider, Heiko Mueller and Peter Hartel
}

CEOS GmbH, Englerstr. 28, D-69126 Heidelberg, Germany

After more than 15 years of having finished the first successful Cs-correction system for a modern high resolution TEM [1] the correction of the spherical aberration is now standard for high resolution electron microscopy. However, the development of aberration correction systems has not been stopped and is continuously going on. The first progress of aberration correction was the conversion from an experimental set-up to a routinely applicable device to be used in commercially available microscopes. One important aspect of this first aberration correction decade was the development of a new series of high resolution TEMs and STEMs dedicated to aberration correction due to an increased overall stability of these microscopes.

Just after the introduction of commercially available Cs-corrected TEM/STEM the discussion started how far one can compensate the resolution limiting aberrations and if one can achieve a new landmark of resolution in the range of $\mathrm{d}=50 \mathrm{pm}$. The chromatic aberration $\mathrm{Cc}$ was the resolution limiting parameter and, hence, had to be cancelled. The benefits of $\mathrm{Cc}$-correction are highest at low energies where the relative energy width DE/E is largest $(\mathrm{E}<100 \mathrm{keV})$. Such a Cc-corrected system has been developed and installed within the TEAM project [2]. The challenging part of this project was not only the electron optics but as crucial the required stability of the power supplies of the correction elements which had to fulfill unprecedented requirements [3]. The requested precision of these voltage and current supplies were assumed to be sufficient for the exposure time of around one second. However, if one assumes the acquisition of focus series for exit wave reconstruction these requirements have to be fulfilled rather for 100 seconds and not only for a short exposure time. Hence, although the stated requirements are fulfilled for single short exposures but a further development of these ultra stable voltage and current supplies is needed to achieve the requested long time stability. Which means an increase of the bandwidth of this ultra stable regime towards lower frequencies. Hence, instead of starting the bandwidth of the relative stability at $1 \mathrm{~Hz}$ it has to achieve the same stability even for $100 \mathrm{~s}$ (from $0.01 \mathrm{~Hz}$ up to $10 \mathrm{kHz}$ ). Which is again a very challenging task and all small disturbances due to temperature changes or mechanical vibrations have to be considered.

The requirements on the stability of the power supplies needed for the compensation of the chromatic aberrations are about one order of magnitude higher than for other strong focusing elements like the objective lens. This is caused by the needed counteracting magnetic and electrostatic quadrupole fields. Roughly $90 \%$ of the strength of the magnetic quadrupole excitation is needed to generate a chromatic aberration constant of negative sign to be used to compensate the $\mathrm{Cc}$ of the objective lens. Hence, the already high stability requirements of an objective lens of around DI/I $\sim 10^{-7}$ is increased to DI/I $\sim 10^{-8}$. When we started the Cc-correction development right from the beginning on we concentrated our efforts on the development of such ultra stable voltage and current supplies. However, one problem when developing such ultra stable supplies is always the precise measurement of the noise, drift and other instabilities and having a comparison when applying such a supply to a real electron beam. With a single supply one never would be able to investigate the stability of a current source, for example, with an electron beam. For this purpose, we developed a dedicated strong dipole element with two windings or two electrodes to measure the instabilities of two supplies against each other. With such a set-up we 
could measure and demonstrate the overall stabilities of the first prototypes.

With the first Cc-corrected TEM the goals with respect of the resolution could be achieved [4]. For the proof of the attained resolution a single image is necessary. However, when using a high resolution TEM and trying to get all information of an unknown object more sophisticated methods are needed for which stable imaging parameters are necessary during the whole acquisition process. Hence, a long time stability of all the supplies is needed for about $100 \mathrm{sec}$ [5]. Such long time stabilities of the power supplies are possible as can be seen by a measurement of a current supply developed for the energy filter of the SESAM Project at the MPI Stuttgart (see Fig. 1) [6].

From the current development of aberration correction systems we have now a good understanding of what can be achieved and what has do be improved to fulfil the requirements of advanced high resolution electron microscopy. The nowadays achieved stabilities can be observed, for example, by the comparison of the measurement of the quadrupole supplies by electronic means and the measurement of the astigmatism within the images over a long time. We found a good agreement of these simultaneously carried out measurements.

In memoriam Professor Gertrude Rempfer

References:

[1] M. Haider et al, Nature, 392, (1998) p 768,

[2] TEAM http://ncem.lbl.gov/TEAM-project/files/TEAM0.5.html

[3] M. Haider et al, Ultramicroscopy 108 (2007) p 178

[4] C. Kisielowski et al., Micr. and Microanal. 14 (2008) p 469

[5] J. Barthel and A. Thust, Ultramicroscopy 111 (2010) p 27

[6] P. van Aken et al., Micr. and Microanal. 13 suppl. 2 (2007) 862 CD

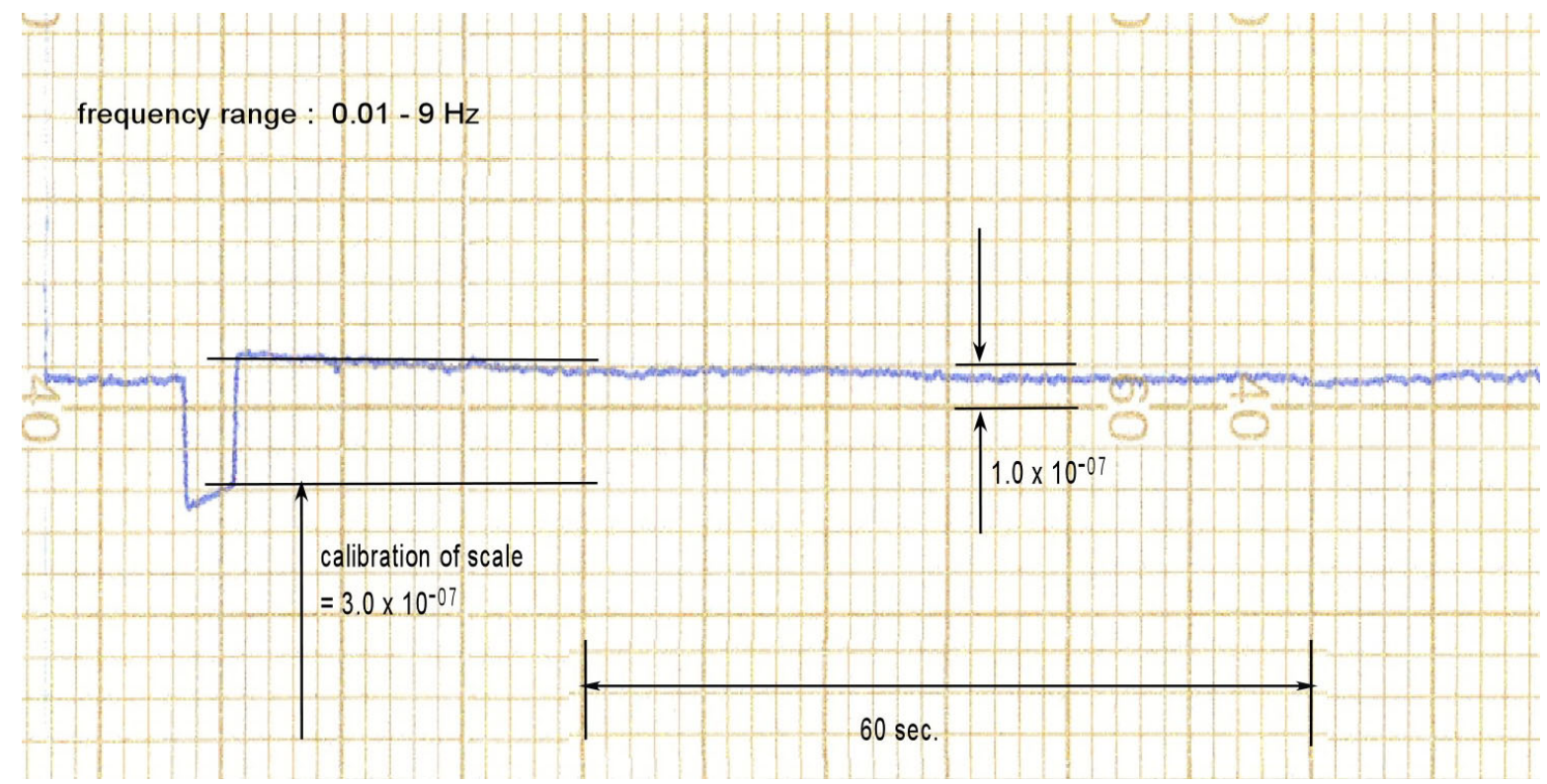

Figure 1: Measurement of the long time stability of the current supply of an energy filter within the frequency range of $0.01 \mathrm{~Hz}-9 \mathrm{~Hz}$. The stability within this frequency range is far below $1.10^{-7}$ (measurement by R. Hoeschen, MPI, Stuttgart, Germany). 